\title{
An Opportunity Cost View of Basestock Optimality for the Warehouse Problem
}

\author{
Nicola Secomandi \\ Tepper School of Business, Carnegie Mellon University, 5000 Forbes Avenue, Pittsburgh, \\ PA 15213-3890, USA, ns7@andrew.cmu.edu \\ Tepper Working Paper 2009-E9 \\ Prepared for Handbook of Integrated Risk Management in Global Supply Chains, P. \\ Kouvelis, O. Boyabatli, L. Dong, and R. Li (eds.)
}

July 2009

\begin{abstract}
This work considers the so called warehouse problem, which is a prototypical problem of the trading activity of a merchant in a commodity market. It is known that the merchant's optimal trading policy for this problem has a basestock structure. The exiting proofs of this result hinge on marginal analysis, and may not be easily accessible to managers. This work provides an elementary derivation of the optimality of this structure relying almost exclusively on geometric arguments based on the notion of opportunity cost of a trade, a concept familiar to commodity merchants. Some aspects of managerial relevance associated with this structure are also discussed. It is hoped that the material presented in this work would be of interest to manages involved in the merchant management of commodity storage.
\end{abstract}

\section{Introduction}

Commodity storage plays a fundamental role in commodity industries (Williams and Wright [14]). The so called warehouse problem is a foundational model of the merchant management of a commodity storage facility. More than sixty years ago, Cahn [3] succinctly introduced this problem in the literature as follows: "Given a warehouse with fixed capacity and an initial stock of a certain product, which is subject to known seasonal price and cost variations, what is the optimal pattern of purchasing (or production), storage and sales?"

In this description, Cahn uses fixed capacity to refer to limited space availability. Thus, this version of the problem is best described as the uncapacitated case; that is, without flow rate limits. In contrast, the capacitated version of the warehouse problem refers to the situation where the warehouse features both space and flow rate constraints. This version of the problem arises in applications such as the merchant management of natural gas storage (Maragos [8], Geman [6], Lai et al. [7]).

In both the uncapacitated and the capacitated cases the optimal policy for the warehouse problem is of the basestock type. That is, there exist two critical inventory levels, the 
basestock targets, such that, during a given time period and for a given spot price, it is optimal to purchase or sell inventory to bring the inventory level as close as possible to these levels when the inventory level is respectively below or above the lower and upper basestock targets; it is optimal to store otherwise (in the uncapacitated case the basestock targets are reachable from every inventory level). In the uncapacitated case this has been shown by Bellman [2], Dreyfus [5], and Charnes et al. [4]; in the capacitated case this has been established, independently, by Rempala [11] in a special case, and by Secomandi [12] in a more general case.

The basestock structure is insightful and characterizes the inventory trading policy computed by methods employed in practice, such as that described by Maragos [8] and studied by Lai et al. [7]. The current literature uses marginal analysis to establish the optimality of this structure. The basic argument behind this type of analysis is the comparison of the marginal cost/revenue associated with an immediate trade with the future (expected) marginal value associated with the resulting inventory level, a basic insight that goes back to Massé [9], as cited by Wallace and Fleten [13]. Although insightful, this literature may not be easily accessible to managers.

The premise of this work is that it is important for managers to understand why the basestock target structure is optimal. This is true whether they are already using methods whose optimal policy has this structure, perhaps without explicitly realizing it, or if they are currently using methods that are not consistent with this structure. Consequently, the goal of this work is to provide a simple derivation of the optimality of the basestock structure almost entirely based on intuitive geometric arguments that rely on the notion of opportunity cost of traded inventory. The familiarity of commodity traders with the concept of opportunity cost, even if only qualitatively, should make the analysis of this work accessible to them.

It is important to emphasize that the analyses available in the literature also rely on a notion of opportunity cost. But the key difference between these analyses and that conducted here is that the latter uses the concept of opportunity cost of a decision, which one could label as total opportunity cost of this decision, while the existing analyses use the concept of marginal opportunity cost. Although these are related concepts, the resulting analyses are different.

The remainder of this work proceeds as follows. Section 2 provides a simple motivating example that illustrates the basestock structure of the optimal policy. Section 3 presents a formal model of the warehouse problem. Section 4 establishes the structure of the optimal 
policy based on the stated opportunity cost argument. Section 5 discusses some managerial aspects related to this structure. Section 6 concludes.

\section{A Simple Motivating Example}

Consider the following simple example discussed in Secomandi [12]. Suppose you are a merchant operating in the wholesale market for a given commodity. For the next three months you have access to a warehouse where this commodity can stored. This facility has a space limit, which is normalized to 1 unit, that is, you can only store a finite amount of commodity, but otherwise one could fill up or empty the facility in a single month.

At the beginning of the first month you can decide to purchase the commodity from the spot market and inject it into the facility during this month. At the beginning of the second month you can purchase additional commodity from this market and inject it into the facility, you can withdraw some of the commodity that you have injected in the previous month and sell it into the wholesale market, or continue to store the commodity that is already in the facility. In the last month you withdraw and sell any amount of commodity available in storage.

To avoid clutter, suppose that the injection and withdrawal marginal costs and the inventory holding cost are zero, and let the discount factor be one. Furthermore, suppose that the price dynamics during the next three months are known to be "medium", "low", and "high," that is there is no uncertainty in these prices, e.g., these could represent futures prices for the next three months as of the first month.

Starting with an empty warehouse, it is optimal to fill up the facility in the second month at the low price, and sell the entire inventory in the third month at the high price. This is a basestock policy with month dependent buy-and-inject (BI) and withdraw-and-sell (WS) basestock levels, specified as follows: In month 1 the BI basestock level is equal to 0 (the minimum space) and the WS basestock level is equal to 1 (the maximum space); in month 2 both the BI and WS basestock levels are equal to 1; and in month 3 they are both equal to 0 . Interestingly, the basestock levels are rather simple, being either equal to 0 or to 1 .

But suppose now that while it is still possible to empty the facility in a single month, filling it up requires more than one month, but less than two months. For concreteness, suppose that you can fill up 2/3-rds of the facility in a single month. You will see that this has a significantly impact on the optimal basestock levels. 
You still want to have a full facility at the beginning of month 3, so that you can withdraw and sell all the available inventory at the high price during this month. You would also like to purchase and inject as much inventory as possible during month 2, at the low price. But given the limit on the injection capacity, you are now forced to purchase and inject some commodity in month 1 , at the medium price. Clearly, in month 1 you would limit yourself to purchase and inject the minimum amount of commodity necessary, which is $1 / 3$, and would purchase and inject the remaining 2/3-rds during month 2.

This is also a basestock policy: in month 1 both the BI and WS basestock targets are equal to $1 / 3$; in month 2 both these targets are equal to 1 (notice that the BI basestock target in this month is not reachable from inventory levels below $1 / 3$ because the injection capacity is $2 / 3$ ); and in month 3 both these targets are equal to 0 . Thus, the effect of limited injection capacity has substantially changed the optimal trading policy. It remains of the basestock type, but one basestock target may not be reachable from some inventory levels, and the basestock targets are no longer always equal to either 0 or 1 .

These example are simple and do not require a formal analysis. But to gain a deeper understanding of why a basestock target policy is optimal, the first step is to formulate a model.

\section{Model}

This section formulates a simplified version of the model presented by Secomandi [12]. This is a finite horizon periodic review dynamic programming model, where inventory trading decisions are made at the beginning of each of a finite number of time periods, each of equal length. It is useful to think of these time periods as the months corresponding to the delivery periods following the maturities of futures contracts traded on the commodity that is being traded.

The main simplification relative to the model of Secomandi [12] is that here the price dynamics are assumed to be deterministic. These prices can be interpreted as the discounted futures prices that are available at the beginning of the time horizon for each relevant maturity. Thus, there is no need to model the discount factor, which is thus taken to be 1 .

These assumptions greatly simplify the exposition, but, as discussed by Lai et al. [7], some heuristic models used in practice are based on periodic reoptimizations of a deterministic model of the type described below. Moreover, when prices evolve stochastically, the basestock 
structure depends on their realizations at each decision epoch, in addition to time.

To avoid clutter, there is also no physical inventory holding cost (the model of Secomandi [12] allows for a proportional holding cost to be incurred in each stage). Set $\mathcal{J}$, defined as $\{1, \ldots, J\}$, where $J$ is an integer greater than 0 , is used to index the time of each inventory trading and operational decision; that is, the $j$-th decision, $j \in \mathcal{J}$, is made at the start of time period $j$. An inventory trading and operational decision is referred to as an action and is denoted by $a$.

A positive action corresponds to a purchase followed by an injection, a negative action to a withdrawal followed by a sale, and zero is the do nothing (DN) action. The trading part of an action is performed at the beginning of each stage, when its payoff is also accounted for. The operational part of an action, that is, an injection or a withdrawal, is executed during a stage, immediately after its associated purchase or sale at the beginning of this stage. This means that inventory injected/withdrawn sold in a given stage is available/unavailable in storage in the next stage.

Let $\left(\hat{s}_{1}, \ldots, \hat{s}_{J}\right)$ be the vector of relevant prices for stages 1 through $J$ (recall that these can be interpreted as discounted futures prices as of stage 1). There are positive marginal costs for withdrawing and injecting the commodity. It is useful to define as $s_{j}$ the marginal cost of buying and injecting one unit of commodity, that is, this is $\hat{s}_{j}$ plus the marginal injection cost. By letting $c$ denote the sum of the marginal withdrawal and injection costs, the net price of withdrawing and selling one unit of commodity is $s_{j}-c$. Thus, the immediate payoff of action $a$ in stage $j \in \mathcal{J}$ is

$$
p_{j}(a):=\left\{\begin{array}{ll}
-\left(s_{j}-c\right) a & \text { if } a<0 \\
0 & \text { if } a=0 \\
-s_{j} a & \text { if } a>0
\end{array}, \forall j \in \mathcal{J}\right.
$$

In each stage, this function is piecewise linear and concave: it has a kink at zero, where its slope decreases from $-\left(s_{j}-c\right)$ to $-s_{j}$.

The warehouse minimum and maximum inventory levels are normalized to 0 and 1 , respectively, so that the feasible inventory set is $\mathcal{X}:=[0,1]$. There are limits to the amount of inventory that can be injected or withdrawn during a stage. These are the injection and withdrawal capacities $\bar{C}>0$ and $\underline{C}<0$, respectively, which are constant across stages. It is assumed that both $\bar{C}$ and $-\underline{C}$ are positive and no larger than 1 . In the uncapacitated case both $\bar{C}$ and $-\underline{C}$ are equal to 1 . In the capacitated case at least one of these quantities is strictly less than 1 ; in the example discussed in $\S 2$, in the capacitated case $\bar{C}=2 / 3$. 
An optimal inventory trading policy for a merchant that controls the warehouse can be obtained by solving the finite horizon dynamic program (1)-(2) formulated below. Set $\mathcal{J}$ indexes the stages and the state space in stage $j$ is $\mathcal{X}$. Denote by $V_{j}(x)$ the optimal value function in stage $j$ and state $x$, which is the sum of all the cash flows accumulated from stage $j$ through stage $J$ under an optimal policy. The dynamic programming recursion is

$$
\begin{aligned}
V_{J+1}(x) & :=0, \forall x \in \mathcal{X} \\
V_{j}(x) & =\max _{a \in[\underline{C} \vee(-x), \bar{C} \wedge(1-x)]} p_{j}(a)+V_{j+1}(x+a), \forall j \in \mathcal{J}, x \in \mathcal{X} .
\end{aligned}
$$

Expression (1) sets boundary conditions. Recursion (2) links the value function across stages and states; notice that $\cdot \wedge \cdot \equiv \min \{\cdot, \cdot\}$ and $\cdot \vee \cdot \equiv \max \{\cdot, \cdot\}$. Moreover, in each stage all the feasible actions are available, but at most one of them is allowed to be chosen optimally. This can be shown to be without loss of generality.

By introducing separate decision variables for the BI and WS actions, it is also possible to formulate model (1)-(2) as a linear program, which is straightforward to solve to optimality with any of the available linear programming solvers. However, the dynamic programming formulation facilitates the analysis of the structure of the optimal policy.

\section{Basestock Optimality}

To illustrate the ensuing results, this section uses a slight variation of the example discussed in $\S 2$ with the following parameter values: $c=0.40, \underline{C}=-1, \bar{C}=2 / 3, J=3, s_{1}=1.20$, $s_{2}=2.20$, and $s_{3}=4.20$. The main difference between this example and the capacitated version of that discussed in $\S 2$ is the presence of nonzero marginal injection and withdrawal costs, reflected in the positive value of $c$ (these marginal costs are both equal to 0.20). The specific values for the prices in each stage are chosen so that the figures illustrated below clearly display what they are intended to convey. However, these prices may not be realistic.

It is useful to begin the analysis of the structure of the optimal policy by pointing out an important property of the optimal value function: in each stage $j$ the function $V_{j}(x)$ is piecewise linear and concave (Secomandi [12]). This function could be linear (weakly concave) in some stage; e.g., $V_{J}(x)=0$ if $s_{J}<c$, and $V_{J}(x)=\left(s_{J}-c\right) x$ if $s_{J}>c$. But in general it is a strictly concave function of inventory, which means that its slope decreases at each of the breakpoints that define it as inventory increases. 


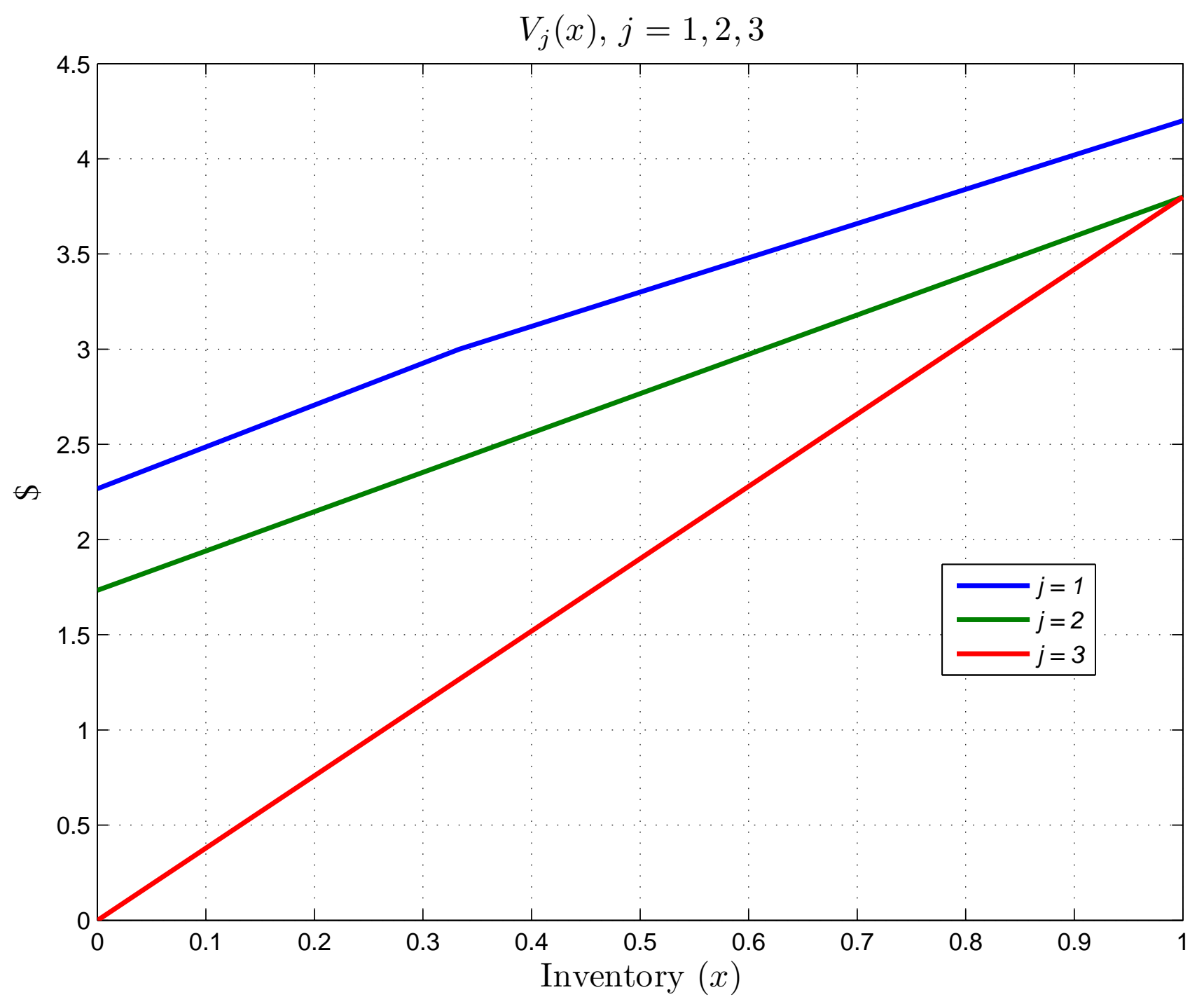

Figure 1: The optimal value function in each stage in the illustrative example. 
Figure 1 illustrates the optimal value function in each of the three stages in the illustrative example. The optimal value function is a straight line in both stages 2 and 3 , and it has a kink at inventory level $1 / 3$ in stage 1 .

In each stage $j \in \mathcal{J}$ and state $x \in \mathcal{X}$, the opportunity cost of a feasible action is the following function, which depends both on the inventory level $x$ and the feasible action $a$ :

$$
O C_{j}(a, x):=V_{j+1}(x)-V_{j+1}(x+a) .
$$

The opportunity cost of a feasible WS action in a given stage and state is the total future value foregone by reducing the available inventory by the corresponding amount. In stage $j$ and state $x$, this value is naturally measured as the difference on the right hand side of (3), that is, the difference between the total future values of entering stage $j+1$ with $x$ and $x+a<x$ units of inventory, respectively.

For a feasible BI action, the concept of opportunity revenue would be more pertinent, i.e., the total future value created by increasing the available inventory by a given amount in a given stage and state. In stage $j$ with inventory level $x$, the opportunity revenue of feasible BI action $a$ would be $V_{j+1}(x+a)-V_{j+1}(x)$. But because the opportunity revenue of such an action is identical to the negative of its opportunity cost, it is sufficient to introduce only the opportunity cost notation.

The opportunity cost of the DN action is identically zero in every stage and state.

The notion of opportunity cost is useful to define the concept of economic action. In stage $j$ with inventory level $x$, a feasible action $a$ is said to be economic if its immediate payoff is equal to or exceeds its opportunity cost: $p_{j}(a) \geq O C_{j}(a, x)$. This definition is intuitive: it simply reflects the fact that it is optimal to perform a feasible action only if its immediate payoff is equal to or exceeds its opportunity cost. In other words, if an action is not economic then it is suboptimal.

According to the definition of economic action, the DN action is economic at every feasible inventory level, because in every stage $j$ it holds that $p_{j}(0)=0$ and $O C_{j}(0, x) \equiv 0$ for every feasible inventory level $x$. Thus, it is useful to interpret the DN action as being both a degenerate WS action and a degenerate BI action; in contrast a negative (respectively, positive) action is referred to as a proper WS (respectively, BI) action.

The following analysis proceeds to show that in each stage the set of feasible inventory levels is subdivided into three regions (subsets), one where only BI actions (both proper and degenerate) are economic, one where only the DN action is economic, that is, no proper BI 
and WS actions are economic, and one where only WS actions (both proper and degenerate) are economic, respectively. This then implies that in each stage only one type of action is respectively optimal in each of these three subsets. More formally, there exist no more than two stage dependent optimal basestock targets $\underline{b}_{j} \leq \bar{b}_{j}$ that subdivide the set of feasible inventory levels $\mathcal{X}$ into the three sets $\left[0, \underline{b}_{j}\right],\left[\underline{b}_{j}, \bar{b}_{j}\right]$, and $\left[\bar{b}_{j}, 1\right]$, where only BI, DN, and WS actions are respectively economic, and hence optimal.

Proceeding in steps, this is now argued geometrically using the notion of opportunity cost in all but the last steps. Figure 2 will be used to illustrate the analysis.

Step 1: Monotonicity of economic actions for a given inventory level. Given inventory level $x$ in stage $j$, consider the opportunity cost as a function of a feasible action $a$. Since the function $V_{j}(x)$ is concave in $x$, it follows from the definition of opportunity cost that that the function $O C_{j}(a, x)$ is convex in $a$ given $x$; also recall that it equals zero at $a=0$. Combining these observations with the fact that the immediate payoff is a concave and continuous function of the action, zero at zero, and decreasing when the action is positive, it is easy to see that the following monotonicity property of economic actions holds: if an action is economic at a given inventory level, then an action with a smaller absolute value but the same sign is also economic at the same inventory level. In other words, if a WS (respectively, BI) action is economic at a given inventory level, then a smaller (in absolute value) WS (respectively, BI) action is also economic at the same inventory level. Figure 2 illustrates these statements in stage 1: specifically, the stated monotonicity in the WS case can be seen by comparing the functions $O C_{1}(a, 1)$ and $p_{1}(a)$; in the BI case, the stated monotonicity can be seen by comparing the functions $O C_{1}(a, 0)$ and $p_{1}(a)$.

Step 2: Mutually exclusive proper economic actions for a given inventory level. Consider a given stage. Given what argued in step 1, it should be clear that if at a given inventory level there exists a feasible proper economic BI (respectively, WS) action, then no feasible proper WS (respectively, BI) action is economic at this inventory level; in the BI case, compare the functions $O C_{1}(a, 1 / 4)$ and $p_{1}(a)$ in Figure 2. Moreover, if only the DN action is economic at a given inventory level, then no proper BI action and no proper WS action are economic at this inventory level; compare the functions $O C_{1}(a, 1 / 3)$ and $p_{1}(a)$ in Figure 2. Hence, at each given inventory level, its corresponding feasible action set is partitioned into a connected subset of economic actions all with the same signs (possibly with the exception of the DN action), and a subset of noneconomic actions.

Step 3: Subdivision of the inventory set into economic BI/DN/WS subsets. It is now of 


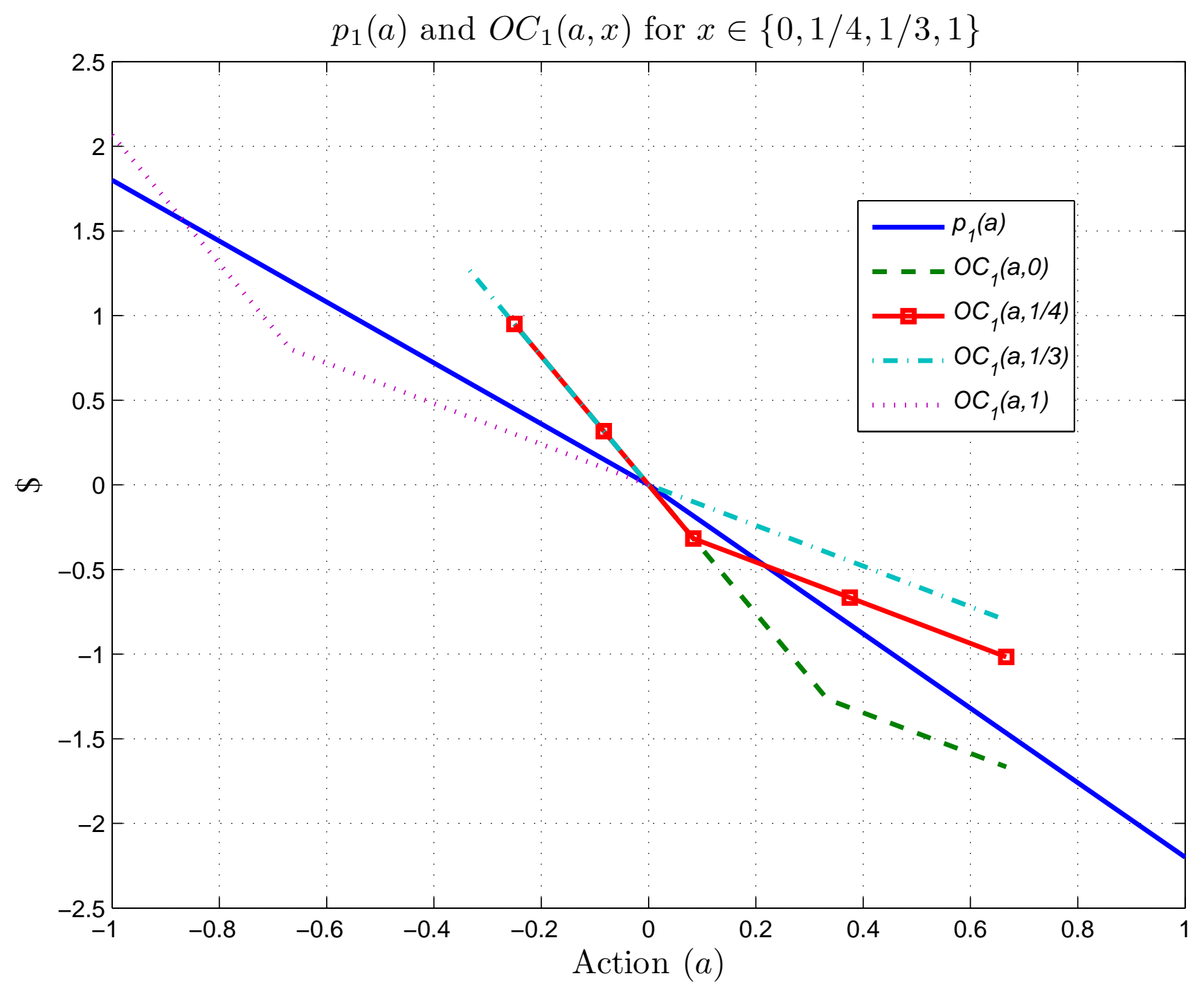

Figure 2: The immediate payoff and the opportunity cost in stage 1 at different inventory levels. 
interest to characterize the behavior of a given economic action when inventory increases in a given stage. To this aim, notice that in each stage the opportunity cost of a given feasible proper WS action (weakly) decreases when inventory increases, while the opportunity cost of a given feasible proper BI action (weakly) increases when inventory increases (assuming that the stated BI action remains feasible when inventory increases). In other words, in each stage the opportunity cost of a given feasible WS (respectively, BI) action rotates counterclockwise around the origin when inventory increases (the domain where the opportunity cost is defined may also change). This should be interpreted in the weak sense, that is, this function may remain constant. Moreover, in the BI case this is true so long as such an action remains feasible when inventory increases. In Figure 2, for proper BI action $a=0.4$ compare functions $O C_{1}(0.4,0), O C_{1}(0.4,1 / 4)$, and $O C_{1}(0.4,1 / 3)$; for proper WS action $a=-0.2$ compare functions $O C_{1}(-0.2,1 / 4)=O C_{1}(-02,1 / 3)$ and $O C_{1}(-0.2,1)$.

Because the immediate payoff function does not depend on inventory, it follows that in each stage if a feasible WS (respectively, BI) action is economic at a given inventory level, then only WS (respectively, BI) actions are economic in this stage at all higher (respectively, lower) inventory levels. Similarly, if only the DN action is economic at two different inventory levels in a given stage, this is also true in this stage at all the inventory levels in between them. But since at every inventory level only one type of action is economic, as argued in step 2, in each stage there exist nonoverlapping inventory subsets that share the same type of economic action and are separated by no more than two inventory levels $\underline{b}_{j} \leq \bar{b}_{j}$. Specifically, only BI actions are economic for $x \in\left[0, \underline{b}_{j}\right]$, only the DN action is economic for $x \in\left[\underline{b}_{j}, \bar{b}_{j}\right]$, and only WS actions are economic for $x \in\left[\bar{b}_{j}, 1\right]$.

Step 4: Subdivision of the inventory set into optimal BI/DN/WS subsets. The last step is to show that the inventory levels that subdivide the feasible inventory set into economic subsets are the optimal basestock targets. This is the only step that requires nongeometric reasoning. Consider stage $j$. Denote by $a_{j}^{*}(x)$ an optimal action in this stage at feasible inventory level $x$.

$\mathrm{DN}$ is the optimal action for each inventory level in set $\left[\underline{b}_{j}, \bar{b}_{j}\right]$, because DN is the only economic action for every inventory level in this set. Thus, it holds that $a_{j}^{*}(x)=0, \forall x \in$ $\left[\underline{b}_{j}, \bar{b}_{j}\right]$.

Consider an arbitrary inventory level $x$ in the region where only BI actions are economic, that is $x \in\left[0, \underline{b}_{j}\right]$. Finding an optimal action for this inventory level entails solving the 
following optimization problem:

$$
\max _{a \in[0, \bar{C} \wedge(1-x)]}-s_{j} a+V_{j+1}(x+a)
$$

This problem can be approached by ignoring the withdrawal and injection capacity limits, by finding an optimal solution $a_{j}^{\diamond}(0)$ to the capacity unconstrained problem

$$
\max _{a \in[0,1-x]}-s_{j} a+V_{j+1}(x+a)
$$

and by setting $a_{j}^{*}(0)$ equal to the minimum between $a_{j}^{\diamond}(0)$ and $\bar{C}$. The capacity unconstrained problem can be simplified by working with the ending inventory $y:=x+a$, rather than an action $a$ and the current inventory level $x$. That is, because $a \equiv y-x$, this problem is equivalent to

$$
\max _{y \in[x, 1]} s_{j} y+V_{j+1}(y)-s_{j} x
$$

Because, by assumption, at $x$ only a BI action or the DN action can be optimal, the constraint $y \in[x, 1]$ can be relaxed to $y \in[0,1]$ without loss of optimality. Thus, the resulting optimization is

$$
\max _{y \in[0,1]} s_{j} y+V_{j+1}(y)-s_{j} x
$$

Further notice that the term $s_{j} x$ in the objective function of this problem does not affect the choice of an optimal solution, and it can be ignored in seeking such a solution. Hence, the relevant optimization reduces to

$$
\max _{y \in[0,1]} s_{j} y+V_{j+1}(y)
$$

An optimal solution to this problem does not depend on which $x$ in the set $\left[0, \underline{b}_{j}\right]$ one is considering. In particular, because DN is optimal for $x=\underline{b}_{j}$, it must be that $\underline{b}_{j}$ is an optimal solution to problem (4). Thus, it holds that the action $\bar{C} \wedge\left(\underline{b}_{j}-x\right)$ is optimal for every $x \in\left[0, \underline{b}_{j}\right]$, that is, $a_{j}^{*}(x)=\bar{C} \wedge\left(\underline{b}_{j}-x\right), \forall x \in\left[0, \underline{b}_{j}\right]$. In other words, inventory level $\underline{b}_{j}$ is an optimal BI basestock target.

It can be argued in a similar manner that inventory level $\bar{b}_{j}$ is an optimal WS basestock target; that is, $a_{j}^{*}(x)=\underline{C} \vee\left(\bar{b}_{j}-x\right)$ for every inventory level $x$ in set $\left[\bar{b}_{j}, 1\right]$. Thus, the optimality of a stage dependent double basestock target structure has been established.

Table 1 displays the optimal basestock targets in each stage for the illustrative example. The optimal policy is identical to that of the example discussed in $\S 2$ in the capacitated case. 
Table 1: The optimal basestock targets in each stage for the illustrative example.

\begin{tabular}{cccc} 
Basestock & \multicolumn{3}{c}{ Stage } \\
\cline { 2 - 4 } Target & 1 & 2 & 3 \\
\hline BI & $2 / 3$ & 1 & 0 \\
WS & $2 / 3$ & 1 & 0 \\
\hline
\end{tabular}

\section{$5 \quad$ Managerial Aspects}

This section discusses some aspects of managerial relevance associated with the basestock structure. The discussion is based on the works of Charnes et al. [4], Bannister and Kaye [1], Maragos [8], Nascimento and Powell [10], Lai et al. [7], and Secomandi [12].

(1) Inventory dependent price characterization. Suppose that in a given stage at least two types of actions are optimal, that is, it does not hold that $\underline{b}_{j}=0$ and $\bar{b}_{j}=1$, in which case the DN action is optimal at all inventory levels, and that $\underline{b}_{j}=\bar{b}_{j}=1$ or $\underline{b}_{j}=\bar{b}_{j}=0$, in which cases filling up and emptying, respectively, the warehouse is optimal at all inventory levels. This implies that the stage $j$ price does not have a unique interpretation: it can be interpreted as being low, intermediate, and high, respectively, at those inventory levels where a BI, DN, and WS action is optimal; only some of these characterizations apply if buying and injecting (respectively, withdrawing and selling) is optimal at some inventory level but withdrawing and selling (respectively, buying and injecting) is not. This means that at a given decision epoch the characterization of a price must be made relative to the merchant's inventory level (Secomandi [12]).

(2) Inventory independent price characterization. This discussion begs the question of whether there exist situations in which the characterization of a price in each stage is inventory independent, that is, if a BI, DN, or WS action is optimal at a given inventory level in a given stage, then the same type of action is optimal at all other inventory levels in the same stage. A situation when this occurs is the uncapacitated case, in which a warehouse can be filled up or emptied in a single stage (Charnes et al. [4]).

Interestingly, this is true even if the immediate payoff function is nonlinear in the action. In the capacitated case, the capacity functions that describe a feasible action as a function of inventory, that is, the quantity $\underline{C} \vee(-x)$ for a withdrawal and the quantity $\bar{C} \wedge(1-x)$ for an injection, are nonlinear in the inventory level $x$. Instead, in the uncapacitated case these functions are linear, being, respectively, $-x$ and $1-x$. Thus, the nonlinearity in the capacity functions in inventory has more profound implications in terms of the parameters 
of the optimal basestock structure than the nonlinearity in the immediate payoff function in the action (Secomandi [12]).

(3) Optimal nontrivial capacity underutilization. In the example discussed in $\S 4$, as well in the capacitated version of that discussed in $\S 2$, in stage 1 it is optimal to underutilize the injection capacity at a nontrivial level. That is, it is optimal to utilize a positive amount of the injection capacity, but not its entirety, whenever BI is optimal, and an analogous statement is true whenever WS is optimal. Thus, the optimal merchant management of a commodity storage facility is far from trivial (Secomandi [12]).

(4) Computation. The double basestock target structure is very useful if one solves problem (1)-(2) by dynamic programming, because only two numbers need to be computed in each stage, that is, the optimal stage dependent basestock targets. In this case, one still faces the issue that the state space is continuous, so that standard backward induction where one computes the value function for each possible stage in each stage is not easily applicable, because there is an infinite number of states in each stage. There are several possibilities to address this issue.

First, one can show that, in addition to being concave, the optimal value function in each stage is piecewise linear. This means that it is characterized by a finite number of slopes. Thus, one can recursively compute the slopes of this function in each stage, from which one can then compute the optimal basestock targets. Bannister and Kaye [1] and Nascimento and Powell [10] propose algorithms that exploit this structure (Bannister and Kaye [1] deal with a variant of the warehouse problem).

Second, if the problem data satisfies a natural "integrality" condition, then the points when the optimal value function may change slope, its breakpoints, can be determined a priori. Specifically, if the injection and the withdrawal capacities and the maximum inventory are all integer multiple of some real number $Q$, then the breakpoints are also so. Then, the only relevant inventory levels that need to be considered in each stage when solving problem (1)-(2) are $0, Q, 2 Q, \ldots, 1$. Secomandi [12] and Lai et al. [7] exploit this result. This is also true in the example previously discussed, where $Q=1 / 3$.

Third, one can solve the problem by linear programming. In this case, one does not obtain a policy for every stage and state, but only the optimal action for each state that is reachable in every stage by following an optimal policy starting from a given initial state.

(5) Stochastic price dynamics. So far, this work has dealt with the case of deterministic price dynamics. In practice, commodity prices are uncertain, e.g., the forward curve of a 
given commodity changes over time. The problem considered here is relevant in practice because it is common among managers to reoptimize a deterministic model similar to the one considered in this work (see, e.g., Maragos [8]). That is, in stage 1 one obtains an optimal policy given the current forward curve and implements the action corresponding to the current state in stage 1. After this action is implemented, one "moves" (in the real world) to the corresponding state at the beginning of stage 2. At this time, one also has available a new forward curve. One can then compute a new optimal policy corresponding to this new forward curve, this is the reoptimization step, and repeat the same procedure.

What this means is that one effectively compute a "slice" of a stage and forward curve dependent double basestock target policy, that is, one in which the basestock targets depend both on the stage and the forward curve in that stage. Because this is done by reacting to the evolution of the forward curve, one computes the basestock targets only for those states that are visited by following such a policy; this is why one only computes a "slice" of this policy, as opposed to the entire policy.

Lai et al. [7] have recently shown, in the context of natural gas storage, that implementing the optimal action obtained from a deterministic policy in a reoptimization fashion generates a nearly optimal policy. Moreover, it is possible to show that the optimal policy when one models the stochastic evolution of the forward curve has also a stage and forward curve dependent basestock target structure.

\section{Conclusions}

This work considers the warehouse problem, a foundational problem in the merchant management of commodity storage. The structure of the problem's optimal inventory trading policy is known to be of the basestock target type. Motivated by the observation that the existing studies of this structure rely on marginal analyses that may not be easily accessible to managers, this work takes a different approach to establish the optimality of this structure. This allows one to proceed almost exclusively by relying on intuitive geometric and arguments based on the notion of opportunity cost. Because the concept of opportunity cost is well known to merchants, if only qualitatively, it is hoped that managers will find this work to be of interest. Specifically, knowledge of this structure is important to them because it could be used to inform their inventory trading decisions. Alternatively, if they are using dynamic or linear programming models to support their inventory trading decisions, their 
decisions are likely consistent with the basestock structure. In this case, this work may provide merchants with an enhanced understanding of their decision making process.

\section{References}

[1] C. H. Bannister and R. J. Kaye. A rapid method for optimization of linear systems with storage. Operations Research, 39:220-232, 1991.

[2] R. Bellman. On the theory of dynamic programming - a warehousing problem. Management Science, 2:272-275, 1956.

[3] A. S. Cahn. The warehouse problem. Bulletin of the American Mathematical Society, 54:1073, 1948.

[4] A. Charnes, J. Drèze, and M. Miller. Decision and horizon rules for stochastic planning problems: A linear example. Econometrica, 34:307-330, 1966.

[5] S. E. Dreyfus. An analytic solution of the warehouse problem. Management Science, 4:99-104, 1957.

[6] H. Geman. Commodities and Commodity Derivatives: Modeling and Pricing for Agriculturals, Metals and Energy. John Wiley \& Sons, Chichester, UK, 2005.

[7] G. Lai, F. Margot, and N. Secomandi. An approximate dynamic programming approach to benchmark practice-based heuristics for natural gas storage valuation. Tepper Working Paper 2008-E38, Tepper School of Business, Carnegie Mellon University, Pittsburgh, PA, USA, 2009.

[8] S. Maragos. Valuation of the operational flexibility of natural gas storage reservoirs. In E. Ronn, editor, Real Options and Energy Management Using Options Methodology to Enhance Capital Budgeting Decisions, pages 431-456. Risk Publications, London, UK, 2002.

[9] P. Massé. Les Réserves et la Régulation de L'Avenir Dans la Vie Économique. Hermann, Paris, 1946. 
[10] J. M. Nascimento and W. B. Powell. Optimal approximate dynamic programming algorithms for a general class of storage problems. Princeton University, Department of Operations Research and Financial Engineering, Princeton, NJ, USA, 2008.

[11] R. Rempala. System Modelling and Optimization, volume 197 of Lecture Notes in Control and Information Sciences, chapter Optimal strategy in a trading problem with stochastic prices, pages 560-566. Springer, Berlin, 1994.

[12] N. Secomandi. Optimal commodity trading with a capacitated storage asset. Management Science, Forthcoming.

[13] S. W. Wallace and S.-E. Fleten. Stochastic programming models in energy. In A. Ruszczynski and A. Shapiro, editors, Stochastic programming, volume 10 of Handbooks in Operations Research and Management Science, pages 637-677. Elsevier, Amsterdam, The Netherlands, 2003.

[14] J. C. Williams and B. D. Wright. Storage and Commodity Markets. Cambridge University Press, Cambridge, UK, 1991. 